\title{
An Automatic Detection by Classification of Cracked Pixels or Noncracked Pixels in Road Surface
}

\author{
Khaddouj Taifi, ${ }^{1}$ Naima Taifi, ${ }^{2}$ Es-said Azougaghe $\mathbb{D}^{\circ},{ }^{2}$ and Said Safi ${ }^{2}$ \\ ${ }^{1}$ Faculty of Sciences and Techniques, Moulay Ismail University, Errachidia, Morocco \\ ${ }^{2}$ Polydisciplinary Faculty, Sultan, Moulay Slimane University, Beni Mellal, Morocco \\ Correspondence should be addressed to Es-said Azougaghe; essaidazougaghe@gmail.com
}

Received 4 May 2021; Revised 24 August 2021; Accepted 3 December 2021; Published 22 December 2021

Academic Editor: Andres Saez

Copyright $(02021$ Khaddouj Taifi et al. This is an open access article distributed under the Creative Commons Attribution License, which permits unrestricted use, distribution, and reproduction in any medium, provided the original work is properly cited.

Automatic detection and monitoring of the condition of cracks in the road surface are essential elements to ensure road safety and quality of service. A crack detection method based on wavelet transforms (2D-DWT) and Jerman enhancement filter is used. This paper presents different contributions corresponding to the three phases of the proposed system. The first phase presents the contrast enhancement technique to improve the quality of roads surface image. The second phase proposes an effective detection algorithm using discrete wavelet (2D-DWT) with "db8" and two-level sub-band decomposition. Finally, in the third phase, the Jerman enhancement filter is usually used with different parameters of the control response uniformity " $\tau$ " to enhance for cracks detection. The experimental results in this article provide very powerful results and the comparisons with five existing methods show the effectiveness of the proposed technique to validate the recognition of surface cracks.

\section{Introduction}

This work concerns the field of metrology for the characterization of the road surface as well as the different geometrical information associated: longitudinal and transversal. This information must be collected regularly in order to effectively program the renovation campaigns. If these repairs are done early enough, they will avoid more work.

One of the criteria used by managers is the surface condition of the roadway. This consists of looking for defects on the pavement such as cracks, joints, and potholes. The most present and most decisive defect to decide on a pavement renovation is the crack. Indeed, these cracks are likely to let the water penetrate deep and deteriorate the structure of the roadway. Cracks can take different orientations or shapes $[1,2]$. They come in longitudinal and transverse and in various fissures. However, the majority of these cracks are either in the axis of the road or perpendicular. The search for defects on the pavement can be presented as the search for break or noise in a regular texture. So, we decided to find stable characteristics of pavement texture that provide different values in the presence of defects. This type of problem is comparable to the search for defects in ceramics [3] or fabrics [4]. Since these textures show less variation than pavement textures, the methods used do not fully address the pavement problem. In some studies [5], the defect is considered as the signal that one tries to detect in noise.

In recent years, we have noticed several studies in industrial processes concerning surface inspection by a computerized system. Because failure of components or surfaces can lead to substantial economic losses, so we must develop an early detection technique of defects and failure prediction. Kordestani et al. [6] presented the state-of-theart and development of failure diagnosis and prognosis (FDP) monitoring systems. Among the methods of surveillance, Rezamand et al. [7] proposed a comparative study of developments for the prognosis of complex critical machines (WT). The objective of the previous topics focused on crack detection; among the crack detection methods, we find the method of structured random forests (RF) [8].

The combination of the approach (RF) method $[8,9]$ and wavelets $[10,11]$ is used to detect the edges of the cracks. 
Amet et al. [12] also presented a method for edge defect detection based on wavelet decomposition and cooccurrence as a descriptor to extract the characteristics of the images to classify the images as defective or nondefective. Mathavan et al. [13] proposed a technique based on the fuzzy transformation of Hough to segment cracks from images of pavements. In the relay metal plate, Aswini et al. [14] used the bottom-hat transform filtering approach and mathematical morphology to detect the surface cracks. Mathematical morphology $[15,16]$ can be used in automatic crack detection system [17] using a process based on JensenShannon divergence and wavelet for detection of the crack. In failure mode classification and health monitoring, Lall et al. [18] proposed a new technique which has been developed based on measured damage precursors. The authors use feature vectors obtained from JTFA for classification. Zalama et al. [19] developed a method based on the Gabor filters to detect the transverse and longitudinal cracks. As a complete method to develop an automatic crack detection system, Chambon and Moliard [20] presented an approach based on a multiscale extraction and a Markovian segmentation addresses the problem of automatic crack detection. Chambon [21] presented a process based in detecting points of interest and a geodesic contour for connecting the points. Nguyen et al. [22] proposed a new approach by applying an anisotropy (FFA) of automatic crack detection. The proposed method using two-dimensional wavelet (2D-DWT) and Jerman enhancement filter gives good performance contrary of the M1 [23] and M2 [20], Gc [21], FFA [22], and MPS [24] methods. Our method is not sensitive to the image texture and produces a very low rate of FN. This paper is organized as follows: Section 2 presents a diagram that contains a method of detecting and extracting cracked pixels or noncracked pixels on the road surface. Section 3 presents the experimental results and analysis. Section 4 gives the concluding remarks. Figure 1 shows the different stages for the development of this system.

\section{Materials and Methods}

2.1. Types of Cracks. There are many types of defects or cracks that must be detected and characterized on the roadways; some examples are illustrated by the images presented in Figures 2-5 [25].

(i) Transversal crack: the transversal crack is perpendicular to the roadway; some examples are illustrated by the images presented in Figure 2 .

(ii) Longitudinal crack: the longitudinal crack is parallel to the roadway; some examples are illustrated by the images presented in Figure 3

(iii) Oblique crack: oblique cracks correspond to slab cracks joining two adjacent sides and are located more than $50 \mathrm{~cm}$ from the corner of the slab; some examples are illustrated by the images presented in Figure 4.

(iv) The various cracks are cracks appearing anarchically on the road surface. There are parabolic cracks, star or Y; some examples are illustrated by the images presented in Figure 5 [26].

(v) Figure 6 shows the road surface without cracks

2.2. Discrete Wavelet Transform. To detect the cracks, we used the wavelets because the latter can be detected in different orientations. More of that are the experimental results by Xiao et al. [27].

The fast algorithm for calculating the wavelet transform was introduced by Mallat in 1989 [28]. This algorithm is known as a two-channel sub-band encoder and it uses quadratic mirror filters. The decomposition of the signal $x$ (n) produces two types of coefficients: approximation coefficients and detail coefficients. These vectors are obtained by the convolution of the input signal with the low-pass and high-pass filters, respectively, followed by a decimation (see Figure 7).

Filters are followed by a decimation by a factor of 2 . The two resulting sequences of column levels will also convolve with the other two banks of identical filters at the first bank of filters to give three sequences of details: diagonal $(\mathrm{HH})$, vertical (LH), and horizontal (HL) and an approximation sequence (LL). Three stages of decomposition are necessary because most of the details are detected by level 3 . The lowest frequency sub-band that is generated is set to zero, since the other sub-bands contain the high frequency information cracks. After this decomposition stage, we then obtain an image that contains only the high frequency information.

We define a family $\Psi_{a, b}(t)$ from the mother wavelet $\Psi$ :

$$
\Psi_{a, b}(t)=\frac{1}{\sqrt{a}} \Psi\left(\frac{t-b}{a}\right)
$$

where $a$ and $b$ are integers representing level and translation, respectively.

$$
C_{a, b}(t)=\frac{1}{\sqrt{a}} \int_{R} f(t) \Psi\left(\frac{t-x}{a}\right),
$$

where $\Psi$ designates the mother wavelet function whose dilated and translated are the bases of a wavelet analysis space on which is projected the function $f(t)$.

\subsection{Enhancement Filters}

2.3.1. Enhancement Function of the Jerman Filter. Enhancement of images is dependent on their relative brightness compared to background; several enhancement filters $[29,30]$ characterize the local structure by analyzing 2nd order. The enhancement function can be developed by redefining the Hessian eigenvalues with respect to the brightness of the structures of interest. Each eigenvalue $\lambda_{i}$; $i=1 ; 2 ; 3$ is redefined as [31]

$$
\lambda_{i}:= \begin{cases}-\lambda_{i}, & \text { bright structures on dark background; } \\ \lambda_{i}, & \text { dark structures on bright background. }\end{cases}
$$

The function of enhancement can be written as 


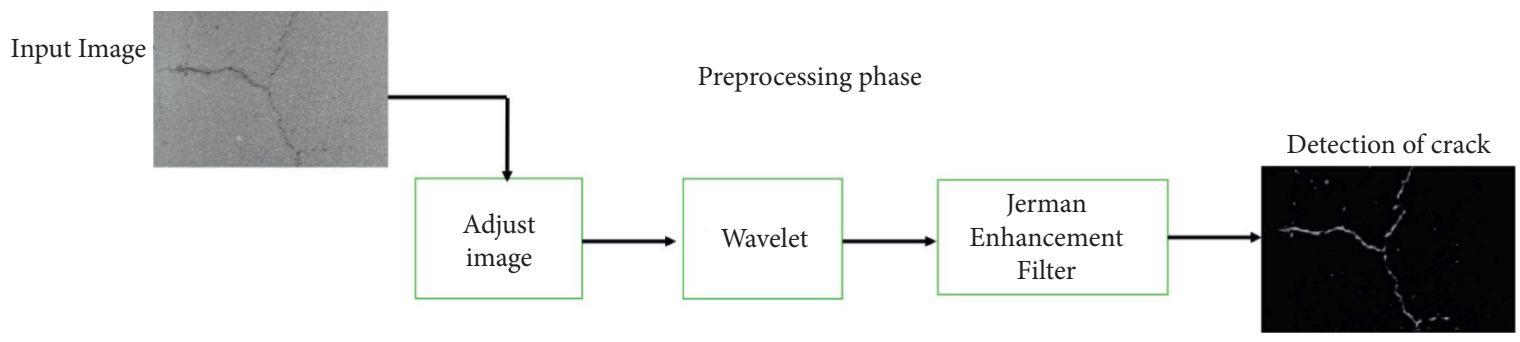

FIGURE 1: The main steps for crack detections.

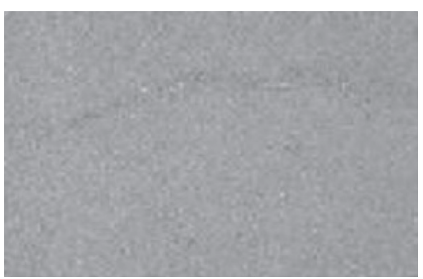

(a)

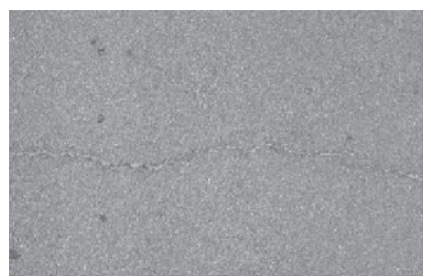

(b)

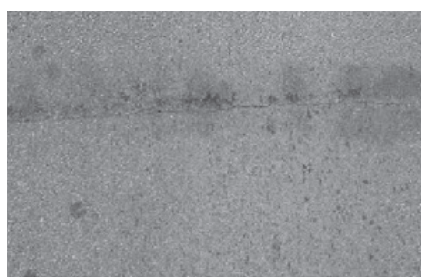

(c)

FiguRE 2: Some defects: (a-c) transversal crack.

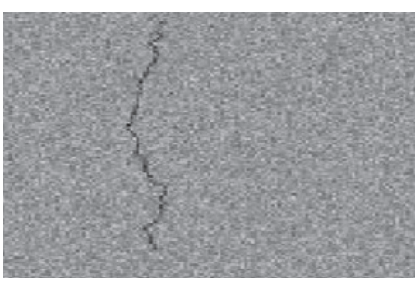

(a)

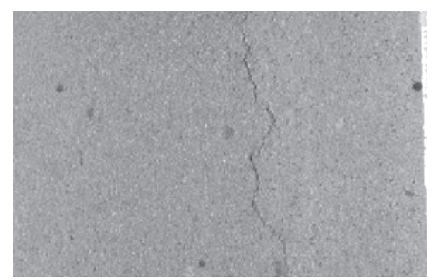

(b)

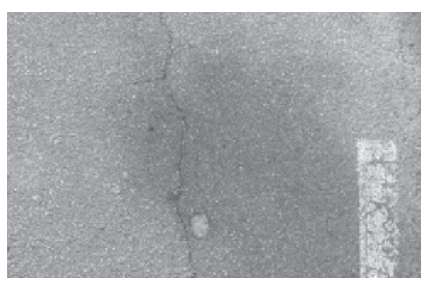

(c)

Figure 3: Some defects: (a-c) longitudinal crack.

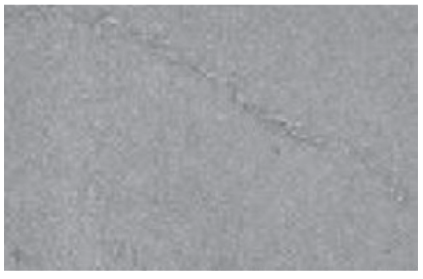

(a)

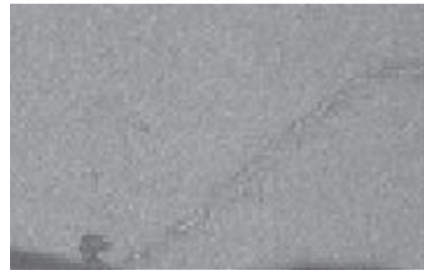

(b)

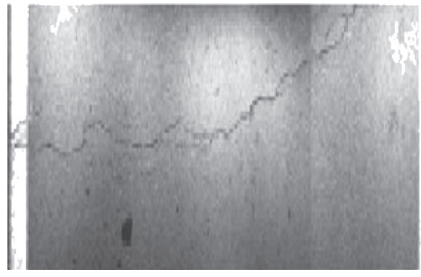

(c)

Figure 4: Some defects: (a-c) oblique cracks.

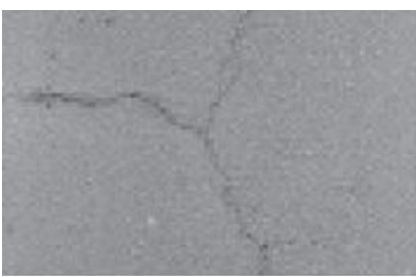

(a)

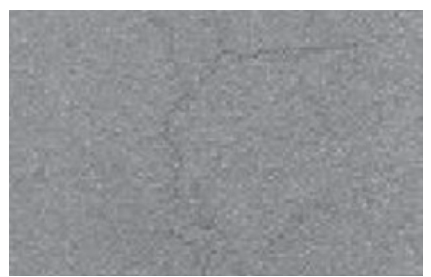

(b)

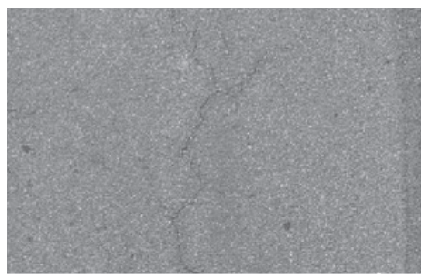

(c)

FIGURE 5: Some defects: (a-c) various cracks. 


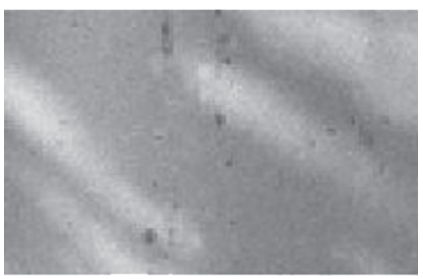

(a)

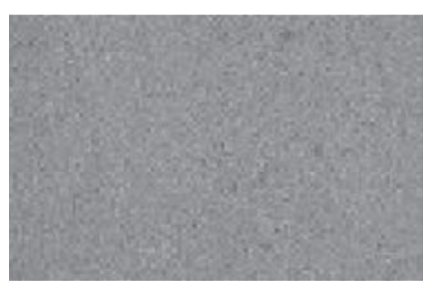

(b)

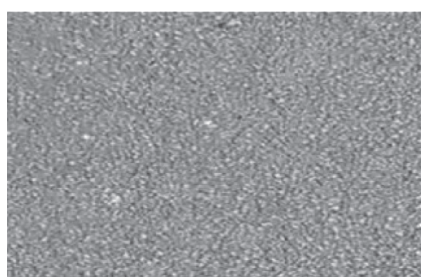

(c)

Figure 6: $(\mathrm{a}-\mathrm{c})$ Normal.

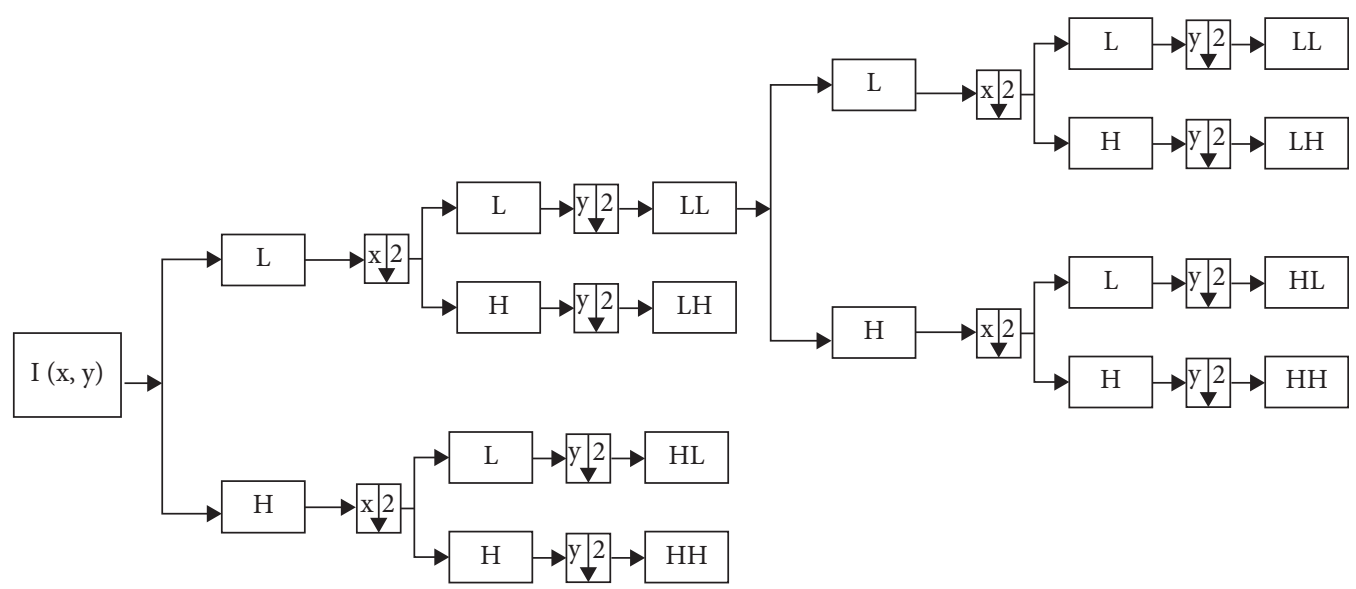

Figure 7: Two-dimensional multiresolution analysis process.

$$
\nu=\lambda_{2}^{2} \lambda_{p}\left[\frac{3}{2 \lambda_{2}+\lambda_{p}}\right]^{3},
$$

Finally, the proposed

$$
v_{p}= \begin{cases}0, & \text { if } \lambda_{2} \leq 0 \vee \lambda_{p} \leq 0, \\ 1, & \text { if } \frac{\lambda_{2} \geq \lambda_{p}}{2>0}, \\ \lambda_{2}^{2} \lambda_{p}\left[\frac{3}{2 \lambda_{2}+\lambda_{p}}\right]^{3}, & \text { otherwise, }\end{cases}
$$

where $\lambda_{p}$ is a parametric regularized version of $\lambda_{3}$, defined to reduce the sensitivity to weakly contrasted region.

$$
\lambda_{p}(s)= \begin{cases}\lambda_{3}, & \text { if } \lambda_{3}>\tau \max _{x} \lambda_{3}(x, s), \\ \tau \max _{x} \lambda_{3}(x, s), & \text { if } 0<\lambda_{3}<\tau \max _{x} \lambda_{3}(x, s), \\ 0, & \text { otherwise. }\end{cases}
$$

\section{Experimental Results and Analysis}

To detect cracks in the road surface image (Figure 8 ), we propose a method that is built in three hybrid stages between them. The first step is the preprocessing of the road surface image to enhance the images of the cracks by adjustment enhancement techniques. In the second step, we use a detection algorithm with wavelet transform (2D-DWT), "db8," and two-level subband decomposition. Finally, the Jerman enhancement filter is usually used for different parameters of the control response uniformity " $\tau$ " to enhance cracks detection.

We studied the behavior of the method by varying the distance " $\tau$ " of the Jerman enhancement filter, the parameter that controls response uniformity. The variation of this distance makes it possible to highlight the nature of the texture and thus makes it possible to filter the "bottom" of the image to reveal only the defects. These images' original inputs (Figure 9) are composed of a background noised uniformly with fault lines of different widths and with different orientations. The tests carried out consisted in varying the distance $\tau$ from 0.5 to 4 of the Jerman enhancement filter. The following images show the values of the proposed method according to the distance $\tau$. In both cases, the defects are perfectly detected. For a distance $\tau$ equal to 4 or higher, the noise has practically disappeared.

These tests, on different types of images, show very good results obtained by our method, provided to correctly choose the parameters including the distance $\tau$ which allows assimilating the nature of the texture.

In the experiment (Figure 10),three detection methods are performed: (b) the LPE [32], (c) Frangi filter, and (d) the proposed robust detection using an effective detection algorithm using discrete wavelet (2D-DWT) with "db8" and two-level sub-band decomposition and Jerman enhancement 

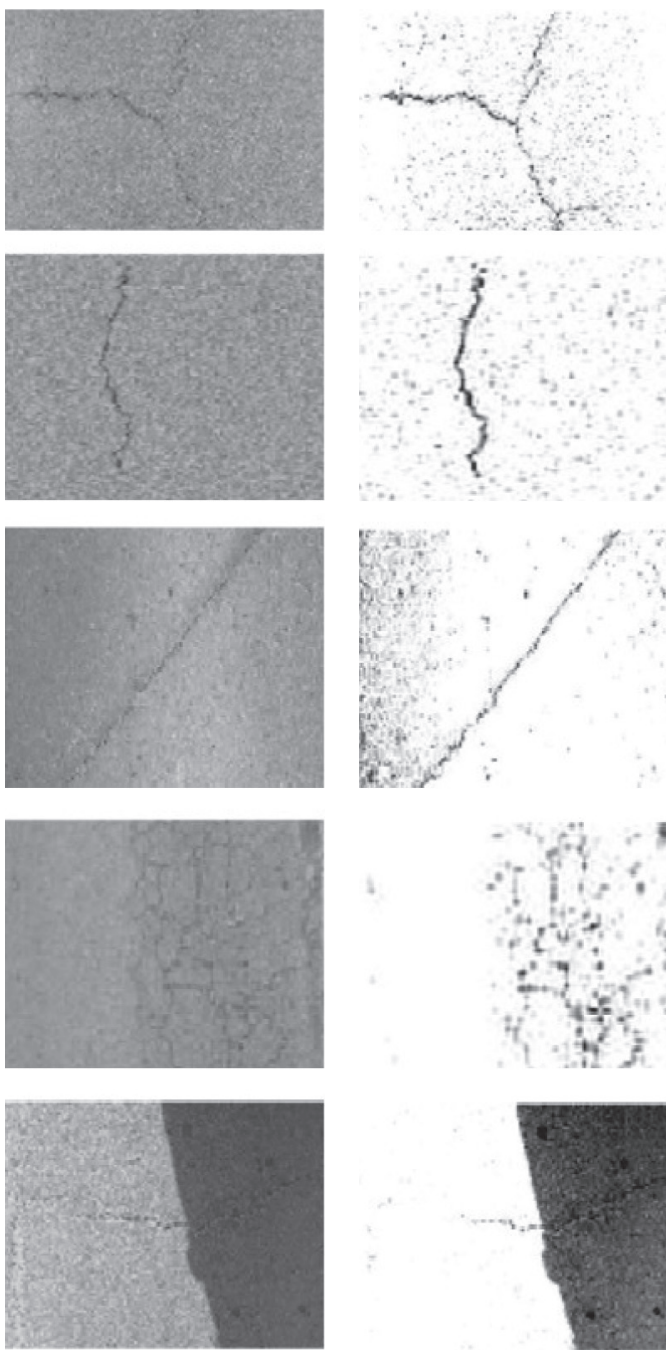

(a)

(b)
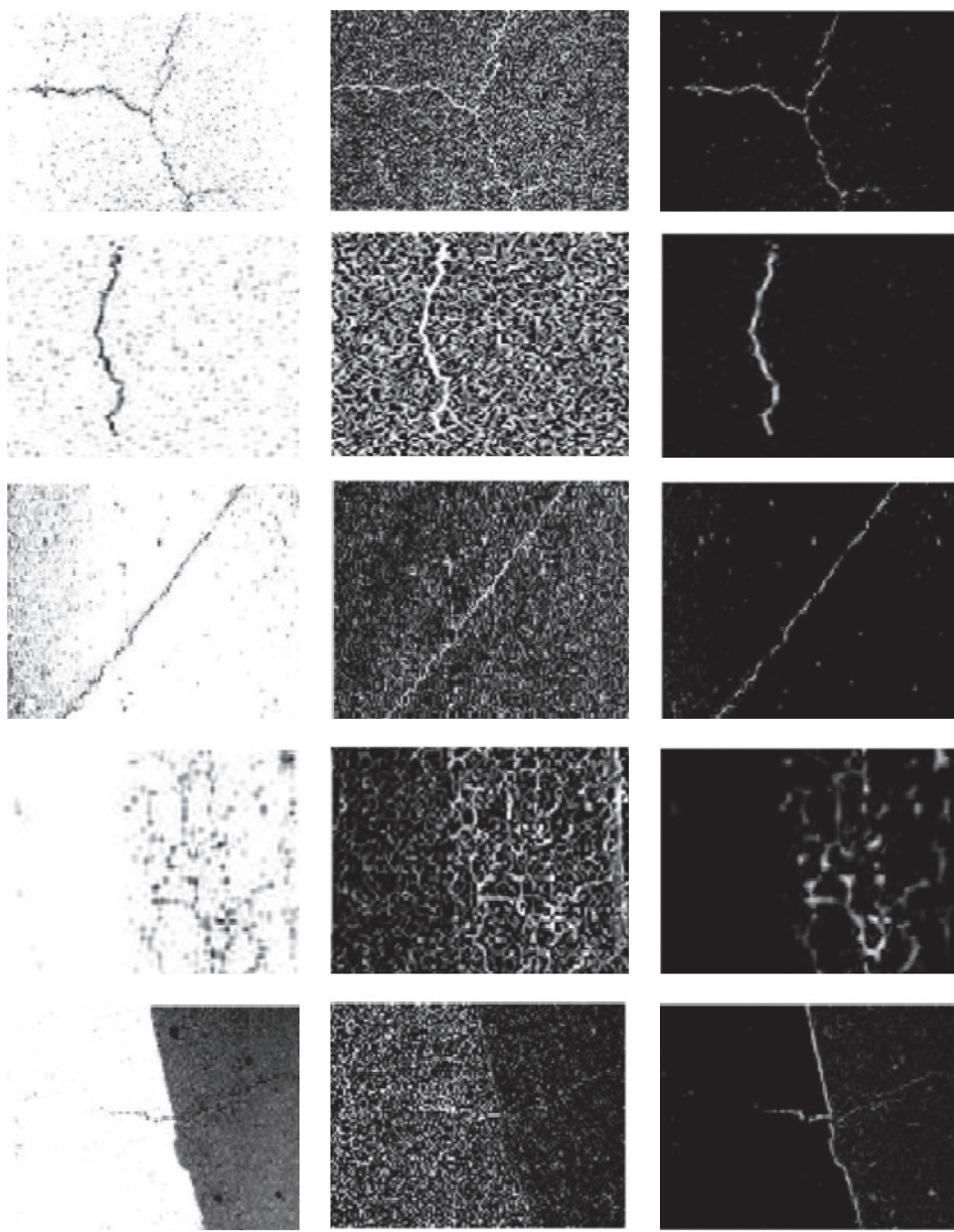

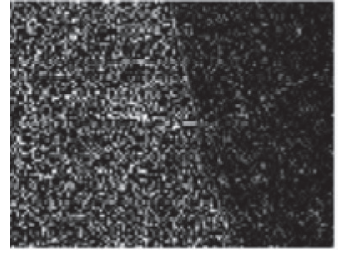

(c)

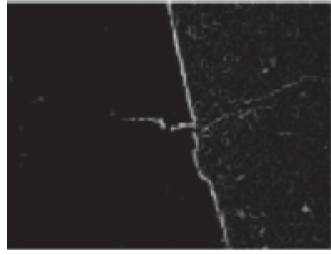

(d)

Figure 8: Preprocessing steps: (a) original image; (b) application of the adjust and DWT; (c) application of the Jerman Filter; (d) application of the proposed method.

Input image
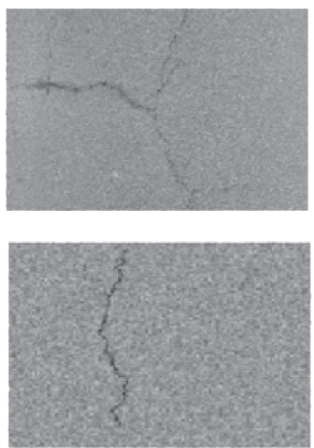

$\tau=0.5$
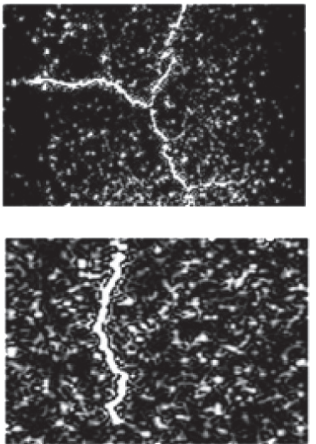

$\tau=1$
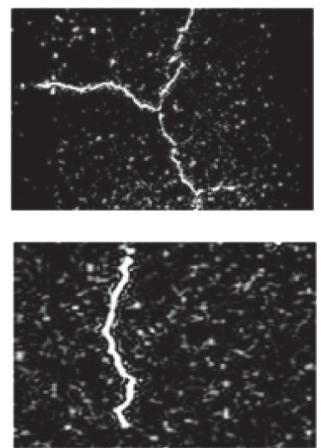

$\tau=4$
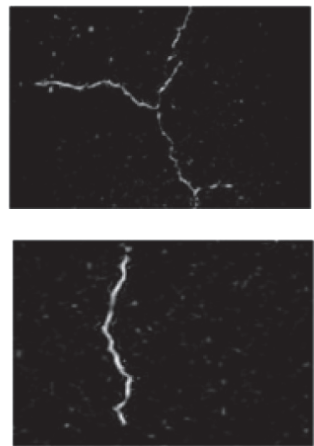

Figure 9: Results of the proposed method according to " $\tau$ " of the parameter that controls response uniformity.

filter. The proposed method aims to eliminate noise and enhance the visibility of cracks in road surface using different parameter of the control response uniformity $\tau$.
LPE: the watershed is the image segmentation tool of mathematical morphology. It has also been studied by Digabel and Lantuéjoul [33] for binary data and then by 

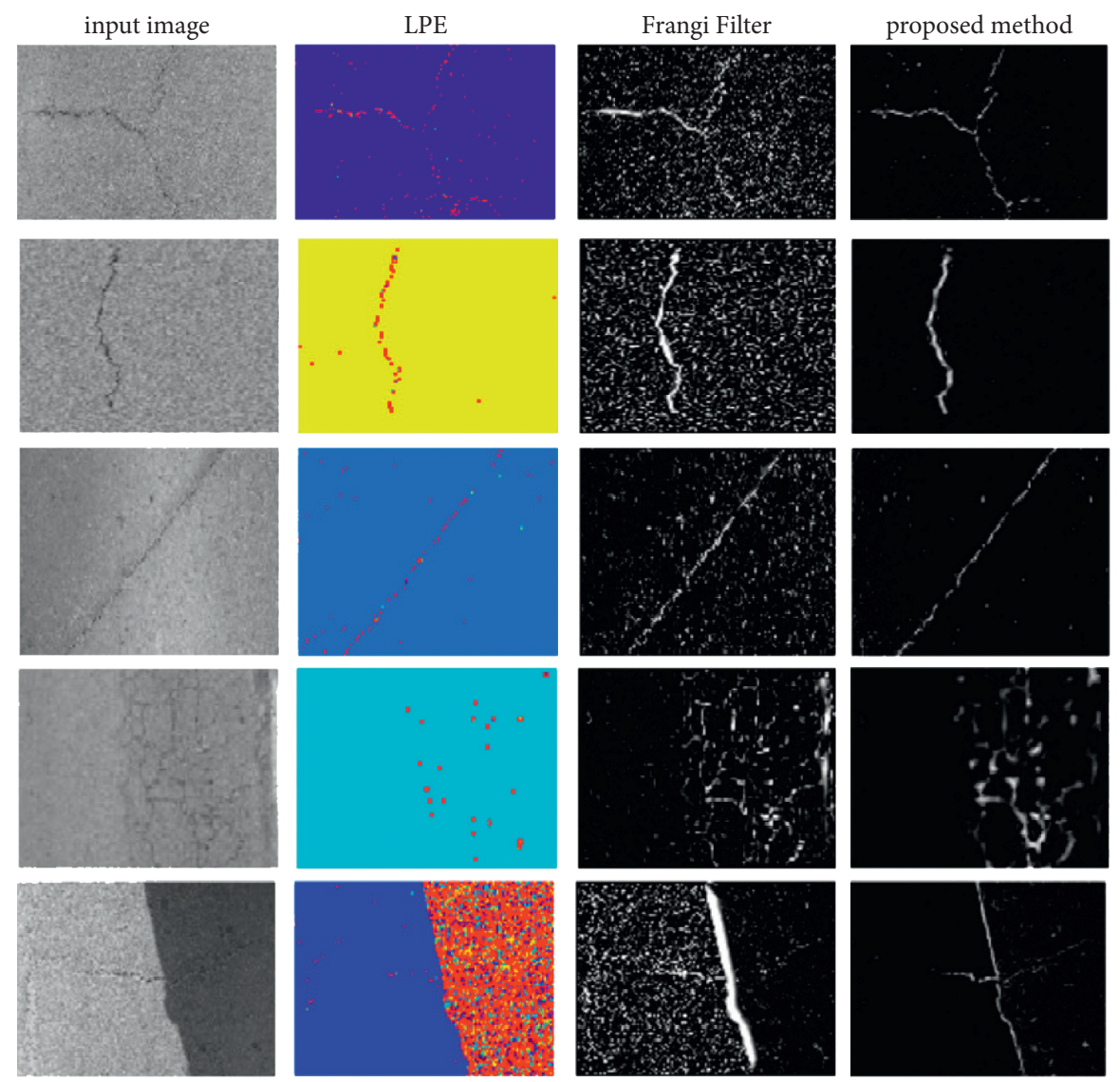

FIGURE 10: Comparison of results with different methods of detection.

Beucher and Lantuéjoul [34] for grayscale image segmentation purposes. The principle of this algorithm is to consider the image as a topographic relief where the luminosity value of each pixel corresponds to a physical elevation. The dark pixels and the light pixels in the roadway image correspond successively to the valleys and hills in the topographic relief [32].

The morphological transformation by LPE then consists in dividing this topographic surface into different basins separated by watershed (LPE) calls for the principle of immersion. The basic idea is to pierce the relief at the level of the capture basins and gradually submerge it in water. At the end of this process, once two retention basins meet, a dam is created to separate them. All of these dams form the watershed (LPE).

3.1. Comparison of Methods. Five cracks detection approaches M1, M2, GC, FFA, and MPS are compared with the proposed method. Figures 11-13 show the crack detection results.

M1 [23] presents a Markovian segmentation preceded by a preprocessing step based on 2D filtering according to multiscale and in four directions to accentuate the contrast. The end result of segmentation is the merging of segmentation results at all levels.
M2 [20] is an improved variant of [23].

GC [21] is a geodesic contour process based on detecting points of interest based on autocorrelation and a geodesic contour for connecting the points.

FFA, free form anisotropy [22], is a method of calculating the minimum paths of each pixel of the image on a block of 30 pixels per side and in four preferred orientations $0,45,90$, and 135 . Then, an anisotropy factor per pixel is calculated from the paths obtained in the previous step. This factor is established from the value of the minimum cost path and the maximum cost path. A large difference between these two values indicates the high probability that the original pixel belongs to a crack.

MPS [24]: this technique, based on a selection algorithm shortest path (Dijkstra), has the effect of promoting the connectivity between the crack pixels. It has two main phases: a segmentation phase, which makes it possible to identify the skeleton of the crack, and a postprocessing phase, which consists in refining the result of the segmentation and estimating the width of the crack.

As Figures 11-13 illustrate, Marlow based methods M1 and M2 demonstrate results of high sensitivity toward the image texture. On the other hand, while GC is evidently 

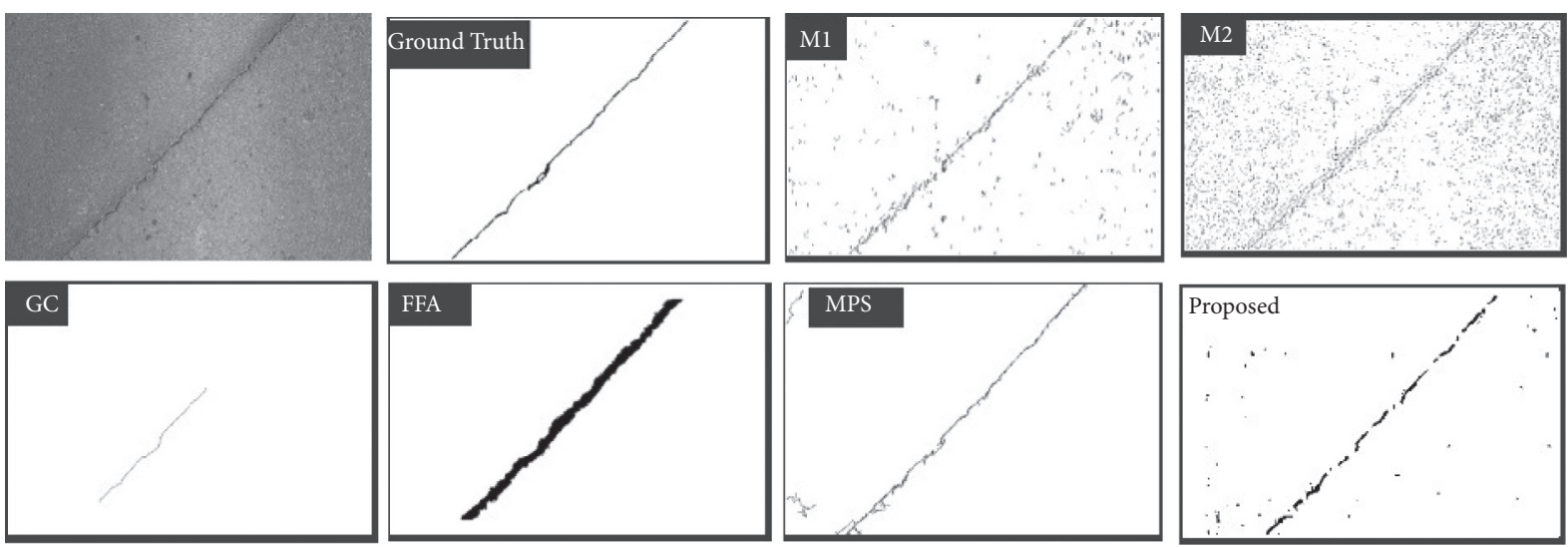

FIGURE 11: Comparing different results generated by different methods of detecting the oblique cracks. M1, M2: 2 Markov-based approaches, M2 is an improved variant of M1; GC: geodesic contours based on points of interest detection; FFA: free form anisotropy based on oriented minimal paths; MPS: minimal path selection and approach proposed in the paper.
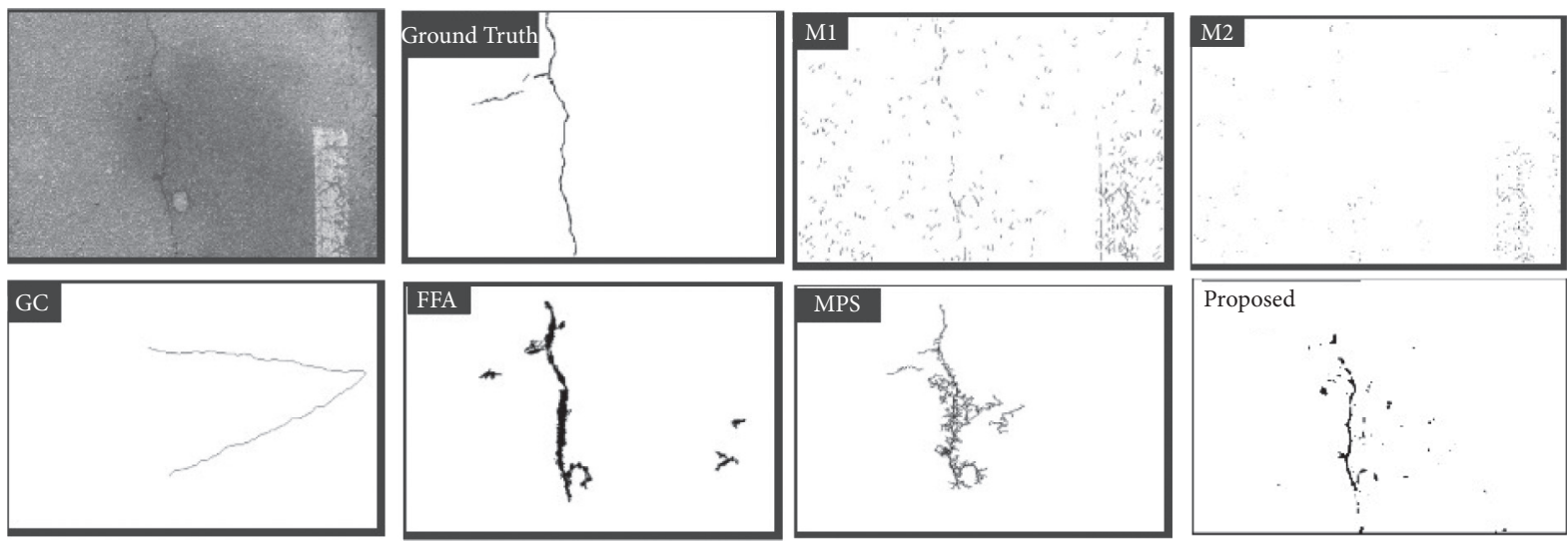

FIGURE 12: Comparing different results generated by different methods of detecting longitudinal crack.
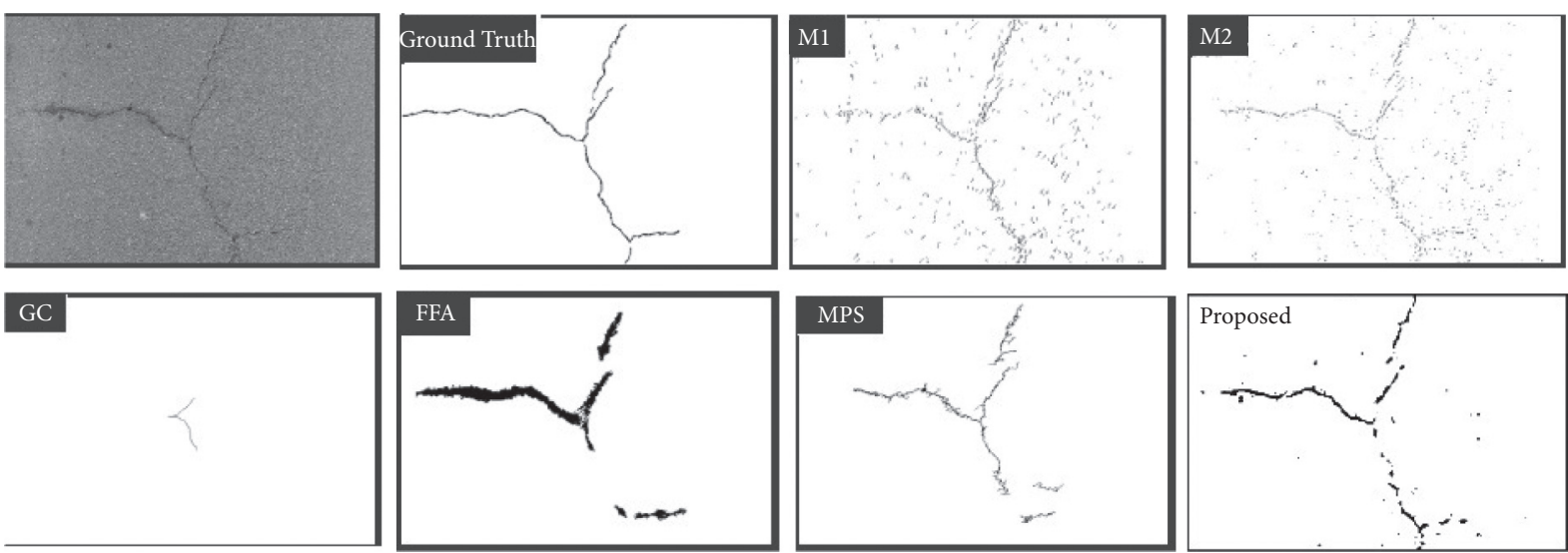

Figure 13: Comparing different results generated by different methods of detecting various crack.

dependent on determining the points of interest automatically, the synthetic image produced by this method is FN due to the fact that this method detects many points of interest but only on a small part of the crack. In contrast, the FFA method is capable of detecting the crack but only if the path of the crack is continuous; otherwise, the chaotic pattern as well as the fine structure of the crack cannot be detected by this method as a result of length and directional constraints. This is clearly manifested in the change of direction which is missed on the right of the thicker crack. 
TABle 1: Confusion matrix.

\begin{tabular}{lcc}
\hline Actual & \multicolumn{2}{c}{ Predicted } \\
\hline & Positive & Negative \\
Positive & TP (true positive) & FP (false positive) \\
Negative & FN (false negative) & TN (true negative) \\
\hline
\end{tabular}

TP: true classification of cracks. FP: false classification of cracks. TN: true classification of noncracks. FN: false classification of noncracks.

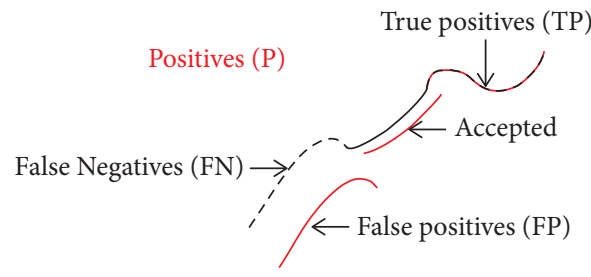

FIGURE 14: Evaluation of the segmentations of the crack.

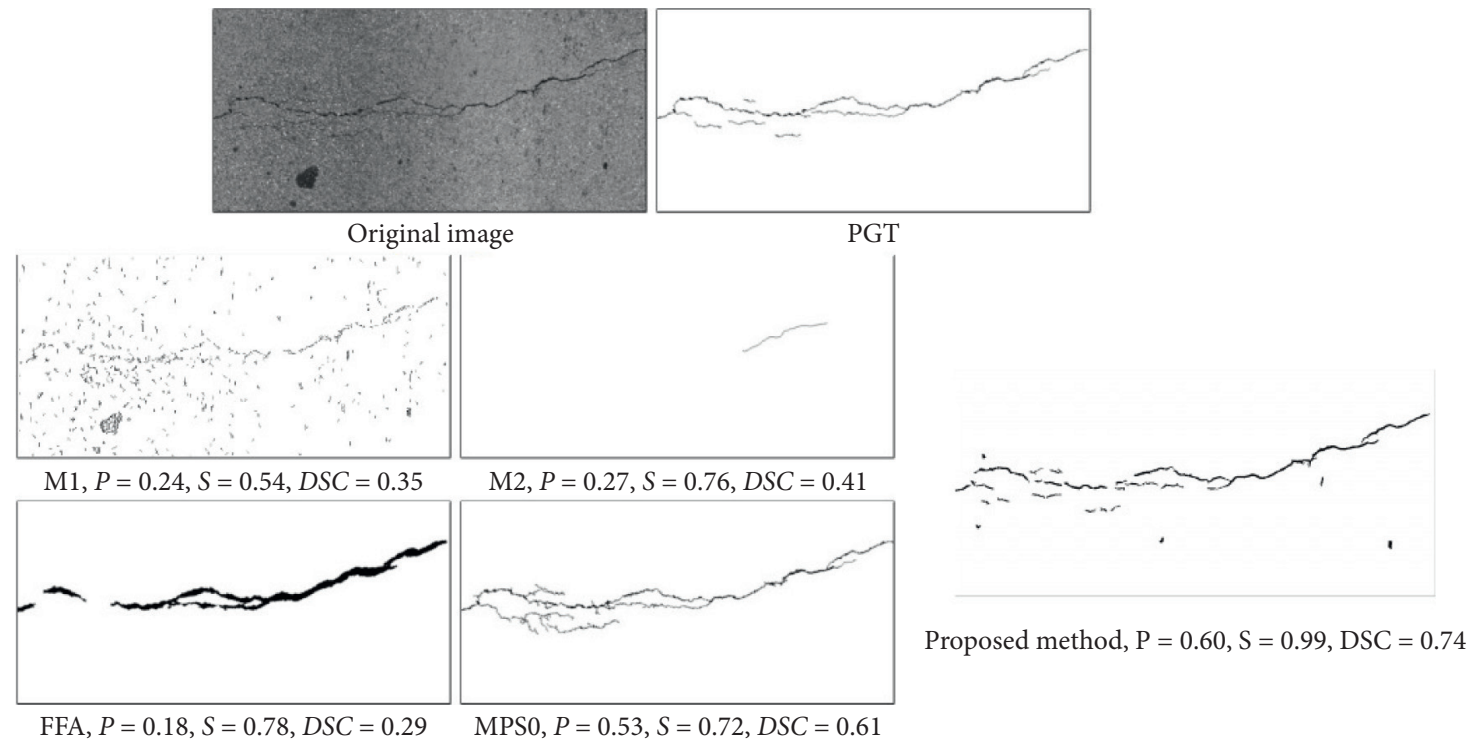

FIGURE 15: Comparison of results with different methods of detection of the transversal crack.

3.2. Performances Evaluation. Consequently, there are four events: two classifications and two misclassifications [35]. The classifications are the true positive (TP) where a pixel is identified as cracks and the true negative (TN) where a pixel is classified as noncracks, the false negative $(\mathrm{FN})$ is negative results in the pixel as cracks, and the false positive (FP) is the positive results in subjects free from cracks.

Table 1 presents the confusion matrix and makes it possible to compare the results obtained by the classifier and the expert.

Figure 14 presents the evaluation of the segmentations of the crack: any pixel is classified each as noncracks or cracks; the black line is a manual (or the ground truth) segmentation and the red line is a proposed segmentation.

Equations (7) and (8) are used to measure the performance metric.
False positives (FP) can be defined as the pixels inside the cracks obtained by the proposed method, but outside the ground truth:

$$
F P=\frac{|P \sqcup R|-|R|}{|R|} \times 100 .
$$

False negative (FN) can be defined as the pixels inside the ground truth, but outside the obtained cracks by the proposed method boundary normalized by the total pixel of the ground truth cracks region.

$$
F N=\frac{|P \sqcup R|-|P|}{|R|} \times 100,
$$

with $P(x, y)$ being the crack region result by the proposed method; $R(x, y)$ is the ground truth. 
In order to assess the performance of classification systems, we have calculated five performance measures which are as follows $[36,37]$ :

The performance parameters (AC, SE, SP, P, and DSC) for cracks segmentation which are presented in equations (6)-(10).

The accuracy (AC)

$$
A C=\frac{T P}{T P+T N+F P+F N} .
$$

The sensitivity $(S)$ is the coefficient which makes it possible to detect the cracks pixels

$$
S=\frac{T P}{T P+F N} .
$$

The specificity (SP) is the coefficient which makes it possible to detect noncracked pixels

$$
S P=\frac{T N}{T N+F P} \text {. }
$$

The precision $(P)[36,37]$

$$
P=\frac{T P}{T P+F P} .
$$

The harmonic mean of precision DICE (DSC) is the coefficient which varies between the best score which is equal to 1 and the bad score which is equal to 0

$$
\mathrm{DSC}=\frac{2 * T P}{2 * T P+F P+F N} .
$$

The results confirm that the proposed method seems better compared to the other methods. The results compared are available from the following web page http://www.irit.fr/ Sylvie.Chambon.

At the level of performance, the proposed method seems better compared to the first four methods. A deep analysis of the result displayed at Figure 15 is evidence that the proposed method detects cracks accurately regardless of the form and thickness with 0.74 in terms of DSC value. Briefly, GC manifests the lowest performance of the tested synthetic image. FA, M1, and M2 provide intermediate results, and the MPS methods also give good performance.

\section{Conclusion}

According different results, it is observed that the proposed method robust detection using effective detection algorithm using wavelet transform (2D-DWT) with "db8" and two-level sub-band decomposition and Jerman enhancement filter improves the visibility of cracks and reduces noise on different types of images, showing very good results obtained by our method, provided to correctly choose the parameters including the distance of " $\tau$ " of the Jerman filter and choose the filter and the discrete wavelet transform. The best results are obtained for $\tau=4$ and "db8" with level $=2[38-41]$.

\section{Data Availability}

The data used to support the findings of this study are available from the corresponding author upon request.

\section{Conflicts of Interest}

The authors declare that they have no conflicts of interest.

\section{References}

[1] T. S. Nguyen, P. Vrignat, M. Avila, S. Bégot, and F. Duculty, "Détection de défauts sur la surface de chaussées par système de vision," GRETSI, 2008.

[2] T. S. Nguyen, M. Avila, S. Bégot, and J. C. Bardet, "Detection of defects in road surface by a vision system," in Proceedings of the The 14th IEEE Mediterranean Electrotechnical Conference, May 2008.

[3] H. Elbehiery, A. Hefnawy, and M. Elewa, "Surface defects detection for ceramic tiles using image processing and morphological techniques," in Proceedings of the Transactions on Engineering, Computing and Technology V5, Istanbul, Turkey, April 2005.

[4] K. L. Mak and P. Peng, "Detecting defects in textile fabrics with optimal gabor filters," Transactions On Engineering, Computing and Technology V13, Springer, New York, NY, USA, 2006.

[5] P. Subirat, "Conception et validation de méthodes de traitement d'images appliquées à la détection de fissures sur les images de surface de chaussées," Thèse de doctorat, Nantes, France, 2006.

[6] M. Kordestani, M. Saif, M. E. Orchard, R. Razavi-Far, and K. Khorasani, "Failure prognosis and applications-A survey of recent literature," IEEE Transactions on Reliability, vol. 70, no. 2, pp. 728-748, 2021.

[7] M. Rezamand, M. Kordestani, C. Rupp, D. S.-K. Ting, and M. E. Orchard, "Mehrdad saif "critical wind turbine components prognostics: a comprehensive review"," IEEE Transactions on Instrumentation and Measurement, vol. 69, no. 12, pp. 9306-9328, 2020.

[8] S. Wang, X. Liu, T. Yang, and X. Wu, "Panoramic crack detection for steel beam based on structured random forests," IEEE Access, vol. 6, 2018.

[9] P. Dollar and C. L. Zitnick, "Fast edge detection using structured forests," IEEE Transactions on Pattern Analysis and Machine Intelligence, vol. 37, no. 8, pp. 1558-1570, 2015.

[10] M. Kordestani, A. A. Safavi, and M. Saif, “A Novel method for harmonic fault diagnosis in power quality system using harmonic wavelet," in Proceedings of the IFAC conference Toulouse, pp. 13569-13574, Toulouse, France, July 2017.

[11] P. Lall, P. Gupta, and M. Kulkarni, "J hofmeister, "time-frequency and autoregressive techniques for prognostication of shock-impact reliability of implantable biological electronic systems"," IEEE Transactions on Electronics Packaging Manufacturing, vol. 33, no. 4, 2010.

[12] L. L. Amet, A. Ertuzun, and A. Ercil, "An efficient method for texture defect detection: subband domain co-occurrence matrices," Image and Vision Computing, vol. 18, no. 6-7, pp. 543-553, 2000.

[13] S. Mathavan, K. Vaheesan, A. Kumar, C. Chandrakumar, K. Kamal, and M. Rahman, "Martyn Stonecliffe-Jones Detection of pavement cracks using tiled fuzzy Hough transform," Journal Electronic Imaging, vol. 26, no. 5, 2017. 
[14] E. Aswini, S. Divya, S. Kardheepan, and T. Manikandan, "Mathematical morphology and bottom-hat filtering approach for crack detection on relay surfaces," in Proceedings of the International Conference Smart Structures and Systems, March 2013.

[15] L. Anders and J. T. Matthew, "Morphology-based crack detection for steel slabs," IEEE Journal of Selected Topics in Signal Processing, vol. 6, no. 7, pp. 866-875, 2012.

[16] R. S. Adhikari, O. Moselhi, and A. Bagchi, "Image-based retrieval of concrete crack properties for bridge inspection," Automation in Construction, vol. 39, pp. 180-194, 2014.

[17] X. Zhang, C. Delpha, and D. Diallo, "Jensen-shannon divergence for non-destructive incipient crack detection and estimation," IEEE Access, vol. 8, 2020.

[18] P. Lall, P. Gupta, and A. Angral, "Anomaly detection and classification for PHM of electronics subjected to shock and vibration," IEEE Transactions on Components, Packaging, and Manufacturing Technology, vol. 2, no. 11, 2012.

[19] E. Zalama, J. Gómez-García-Bermejo, R. Medina, and J. Llamas, "Road crack detection using visual features extracted by Gabor filters," Computer-Aided Civil and Infrastructure Engineering, vol. 29, no. 5, pp. 342-358, 2014.

[20] S. Chambon and J. M. Moliard, "Automatic road pavement assessment with image processing," International Journal of Geophysics, vol. 2011, Article ID 989354, 20 pages, 2011.

[21] S. Chambon, "Detection of points of interest for geodesic contours: application on road images for crack detection," in Proceedings of the International Joint Conference on Computer Vision Theory and Applications, Vilamoura, Portuga, March 2011.

[22] T. S. Nguyen, S. Begot, F. Duculty, and M. Avila, "Free-form anisotropy:A new method for crack detection on pavement surface images," ", IEEE, in Proceedings of the IEEE International Conference on Image Processing, September 2011.

[23] P. S. Chambon and J. Dumoulin, "Introduction of a wavelet transform based on 2D matched filter in a Markov random field for fine structure extraction: application on road crack detection," in Proceedings of the IST/SPIE Electronic Imaging Image Processing: Machine Vision Applications II, San Jose, CA, USA, February 2009.

[24] S. C., J. I. Amhaz and V. Baltazart, "A new minimal path selection algorithm for automatic crack detection on pavement images," in Proceedings of the International Conference on Image Processing, Paris, France, October 2014.

[25] H. Salam, Détection automatique de fissures dans des images de chaussée par modélisation markovienne, Institut de Recherche en Communications et Cybernétique de Nantes, IRCcyn UMR CNRS 6597, Nantes, France, 2010.

[26] Method d'essai no. 52, catalogue des dégradations de surface des chaussées". rapport general, Laboratoire Central des Ponts et Chaussées (LCPC), Paris, France, 1998.

[27] X. Xiao, G. Tian, D. Liu, M. Robinson, and A. Gallagher, "Developments in GPR Based NDT for Ballastless Track of High-Speed Railways," in Electromagnetic Non-destructive Evaluation (XXIII)Amsterdam, Netherlands, 2020.

[28] S. G. Mallat, "Multifrequency channel decompositions of images and wavelet models," IEEE Transactionnnnnnn, vol. 37, no. 12, 1989.

[29] Q. Li, S. Sone, and K. Doi, "Selective enhancement filters for nodules, vessels, and airway walls in twoand three-dimensional CT scans," Medical. Physics, vol. 30, pp. 2040-2051, 2003.

[30] C. Lorenz, I.-C. Carlsen, T. M. Buzug, C. Fassnacht, and J. Weese, "A multi-scale line filter with automatic scale selection based on the hessian matrix for medical image segmentation," in Scale-Space Theory in Computer Vision, B. t. H. Romeny, L. Florack, J. Koenderink, and M. Viergever, Eds., Springer, Berlin, Germany, Jan. 1997.

[31] T. Jerman, F. Pernus, B. Likar, and Z. Spiclin, "Enhancement of Vascular Structures in 3D and 2D Angiographic Images," IEEE Transactions on Medical Imaging, vol. 35, no. 9, 2016.

[32] L. Vincent and P. Soille, "Watersheds in digital spaces: an efficient algorithm based on immersion simulations," IEEE Transactions on Pattern Analysis and Machine Intelligence, vol. 13, no. 6, pp. 583-598, 1991.

[33] H. Digabel and C. Lantuéjoul, "Iterative algorithms," in Proceedings of the 2nd European Symposium Quantitative Analysis of Microstructures in Material Science, Biology and Medicine, pp. 85-99, Riederer Verlag, 1978.

[34] S. Beucher and C. Lantuejoul, "Use of watersheds in contour detection dans," in Proceedings of the International Workshop on Image Processin, Real-Time Edge and Motion Detection/ Estimation, pp. 17-21, Rennes, France, septembre 1979.

[35] M. Mousavi, M. Moradi, A. Chibakhsh, M. Kordestani, and M. Saif, "Ensemble-based fault detection and isolation of an industrial gas turbine," in Proceedings of the IEEE International Conference on Systems, Man and Cybernetics (SMC), Toronto, Canada, October 2020.

[36] L. Houam, Contribution à l'analyse de texture de radiographies osseuses pour le diagnostic pr è coce de l'ost è oporose, These Universite d'Orleans, Orléans, France, 2013.

[37] K. Veropoulos, "Machine Learning Approaches to Medical Decision Making, PhD thesis, University of Bristol, Brstol, England, 2001.

[38] R. H. Bamberger and M. J. T. Smith, "A filter bank for the directional decomposition of images: theory and design," IEEE Transactions on Signal Processing, vol. 40, no. 4, pp. 882-893, 1992.

[39] O. Boiman, E. Shechtman, and M. Irani, "In defense of nearest neighbor based image classification," in Proceedings of the IEEE Conference on Computer Vision and Pattern Recognition, June 2008.

[40] J. Kopp, "Efficient ndh $3 \times 3$ matrices," International Journal of Modern Physics C, vol. 19, no. 03, pp. 523-548, 2008.

[41] M. Vetterli and O. Rioul, "Wavelet and signal processing," IEEE Signal Processing Magazine, vol. 8, no. 4, 1991. 